\title{
Proprioceptive Influences on Auditory and Visual Spatial Localization ${ }^{1}$
}

\author{
JAMES R. LACKNER ${ }^{2}$ AND BARBARA SHENKER \\ Ashton Graybiel Spatial Orientation Laboratory, Brandeis University, Waltham, Massachusetts 02254
}

\begin{abstract}
We evaluated the influence of proprioceptive information about arm position on the perceptual localization of auditory and visual targets attached to the hand. Our approach was to distort the perceived position of the restrained arm by means of mechanical vibration of the biceps brachii muscle; such vibration elicits compelling apparent extension of the stationary forearm (Goodwin, G. M., D. I. McCloskey, and P. B. C. Matthews (1972) Science 175: 1382-1384, Brain 95: 705-748), and subjects report changes in the apparent directions of the auditory and visual targets attached to their hand. These changes are in the same direction and plane as apparent arm motion and their onsets are coincident with or lag slightly behind the experienced displacement of the arm. While visual motion is being experienced, a subject's eyes remain steadily fixating the target light. The pattern of findings demonstrates that (1) proprioceptive information about limb position can influence the central representation of gaze and (2) auditory localization can be similarly influenced. The biasing of auditory localization indicates that identical patterns of arrival time and intensity cues at the two ears can give rise to the perception of sounds in widely disparate spatial positions in relation to the head and body, depending on the proprioceptive representation of the direction of the sound source.
\end{abstract}

Sherrington $(1900,1906)$ believed that the spindle receptors within striated muscle contribute to conscious position sense and kinesthesia. He based his conclusions in part on the observation that the number of myelinated muscle afferents associated with a musclecontrolling movement about a joint is large in relation to the number of myelinated joint afferents, and in part on speculations concerning the possible role of extraocular muscle spindles in the position sense of the eye. Until relatively recently, Sherrington's conclusions were disputed and kinesthetic sensibility was attributed to receptors of the joints (for extensive reviews of this debate, cf. Goodwin et al., 1972c; Granit, 1972; Matthews, 1972, 1982; McCloskey, 1978, 1981).

Several recent investigations, however, have reaffirmed the validity of Sherrington's $(1900,1906)$ conclusions. Burgess and Clark $(1969 \mathrm{a}, \mathrm{b})$ and Clark and Burgess (1975) have recorded from dorsal root filaments in articular nerves from the cat knee joint, exploring the relationship between filament activity and the full range of knee

Received February 6, 1984; Revised September 12, 1984;

Accepted September 14, 1984

\footnotetext{
${ }^{1}$ Support was provided by the National Aeronautics and Space Administration, Contract NAS 9-15147.

${ }^{2}$ To whom correspondence should be addressed.
}

motion. Their findings suggest that these filaments cannot contribute useful information about knee joint angle in any simple and direct fashion. Granit (1972), who has analyzed errors in the appreciation of movement that result from interfering with or obstructing the course of voluntary movements, has argued forcefully that muscle spindles participate in position sense and that "unexpectedly" high levels of spindle discharge are associated with misjudgments of muscle length. Goodwin et al. (1972a, b, c), in an extensive series of experiments, demonstrated a profound influence of muscle spindie activity on apparent limb orientation. They showed that, if the motion of a limb moving under the action of a tonic vibration reflex (vibration of a skeletal muscle activates its primary and secondary spindle endings, leading to its reflex contraction and inhibition of its antagonist-a phenomenon that is known as the tonic vibration reflex; Hagbarth and Eklund, 1966) is resisted, then illusory motion of the stationary, unseen limb will result. The direction of experienced motion is like that which would be associated with stretch of the vibrated muscle; e.g. vibration of the biceps brachii elicits reflex movement of the forearm into flexion, and resisting this motion leads to experienced extension of the stationary forearm.

Lackner and Levine (1979), extending the findings of Goodwin et al. $(1972 a, b, c)$, found that by vibrating the appropriate postural muscles, apparent motion of the stationary body could be induced in virtually any desired direction. Such experienced motion was often accompanied by reflexive oculomotor compensations and other postural reflexes. In their studies, somatosensory information about body orientation also influenced the way in which particular patterns of muscle vibration affected the ongoing apparent orientation of the body; the experienced body orientation was always one that was consistent or meaningful in terms of the conjoint patterns of stimulation.

In related studies, Lackner and Levine (1978) found that if a target light was attached to a subject's hand and illusory motion of his forearm was induced by vibration of the biceps or triceps brachii muscle, the subject would see the physically stationary target light move in the direction of illusory arm motion and by nearly the same amount. Moreover, while a subject was perceiving motion and displacement of the target, his eyes would remain steadily fixating the target light. Lackner and Levine (1978) concluded that during such an "oculobrachial illusion" the subject's stationary eyes were centrally interpreted as in motion because, otherwise, a retinally stable target should not have been perceived as undergoing motion-unless, of course, the subject had perceived his head, or head and body, as also in motion, which was not the case.

Levine and Lackner (1979) found that the magnitude of illusory arm displacement induced by muscle vibration was diminished when a target light was attached to the hand. This observation indicates that, just as spatial information about arm position can influence the representation of eye position, so, too, can oculomotor information affect the perception of limb perception. Such bidirectional influences imply that, although there are separate and distinct central nervous system specifications of arm and eye positions, at some 
level of analysis these representations are nevertheless correlated and interrelated in terms of a supraordinate representation of body orientation involving the configuration of the entire body.

The prosont study represents an attempt to explore further the character of these supraordinate representations through the analysis of intersensory interactions involving proprioception, vision, and audition. With regard to audition, we were interested in the extent to which proprioceptive information about the spatial location of an auditory target would affect its apparent position. The perceived spatial position of a sound source in relation to a listener and his environment is known to depend on the arrival times and acoustic characteristics of the stimuli at his two ears (von Bekesy, 1960), representations of head-trunk position, and whether the body is registered as in motion (Wallach, 1939, 1940; Graybiel and Niven, 1951; Lackner, 1973, 1983; Lackner and Graybiel, 1978a, b, 1979). We wished to determine whether a sound source attached to the stationary hand would be heard as moving and changing position when illusory motion of the hand was evoked by muscle vibration. An important aspect of this test situation is that, in humans, the ears, unlike the eyes, are rigidly affixed to the head and do not undergo independent directional changes. Consequently, if auditory motion is to be induced by proprioceptive misinformation about the location of an auditory target, then several perceptual outcomes are likely: the subject might feel his physically stationary head moving on his torso in keeping with the change in apparent position of the auditory source; he might hear the sound source as changing location in relation to his head although the auditory cues at his ears are unchanging; or he might experience a dissociation between perceived auditory movement and displacement. The latter alternative is also characteristic of the oculobrachial illusion: the target light will initially be seen as moving and displacing; then the target light, while still seeming to move, will no longer be perceived as displacing (Lackner and Levine, 1978). Dissociations of apparent movement and displacement are also a feature of the oculogyral illusion (cf. Graybiel and Hupp, 1946).

The present experiment studied the influence of vibratory myesthetic illusions ${ }^{3}$ on auditory and visual localization using two orientations of the arm. These orientations were chosen so that illusory motion of the arm induced by vibration could be confined to a horizontal or to a vertical plane. If a sound source is physically displaced within a listener's median plane, the arrival time and intensity cues at the two ears always remain equal; by contrast, when a sound source is displaced in a horizontal plane, the arrival time and intensity cues at the ears become asymmetric. Accordingly, it is physically possible to have auditory movement in the median plane without alteration of the time and intensity cues at the two ears but not auditory movement in a horizontal plane. We expected, therefore, that it might be easier in our experimental situation to induce apparent auditory displacement in the median rather than in a horizontal plane because the physical cues at the two ears would always be the same and constant.

\section{Materials and Methods}

Subjects. Eight volunteers, ages 18 to 28 years, with normal hearing and without known oculomotor deficits or motor impairments participated. They had been pretested to ensure that they experienced vibratory myesthetic illusions during vibration of the biceps brachii and triceps brachii muscles. Four had taken part in prior experiments involving vibratory myesthetic illusions. All were experienced in making psychophysical judgments.

Apparatus. Two separate apparatus were used, one for apparent movement of the forearm in a vertical plane and one for the horizontal plane. In both apparatus, the subject was seated with his head stabilized by an individually molded biteplate. For the vertical condition, the subject's right arm was strapped into a contoured, padded support that fixed the elbow at

${ }^{3}$ Lackner and Levine (1979) introduced the term, vibratory myesthetic illusion, to refer to the general category of vibration-induced illusions of muscle lengthening.

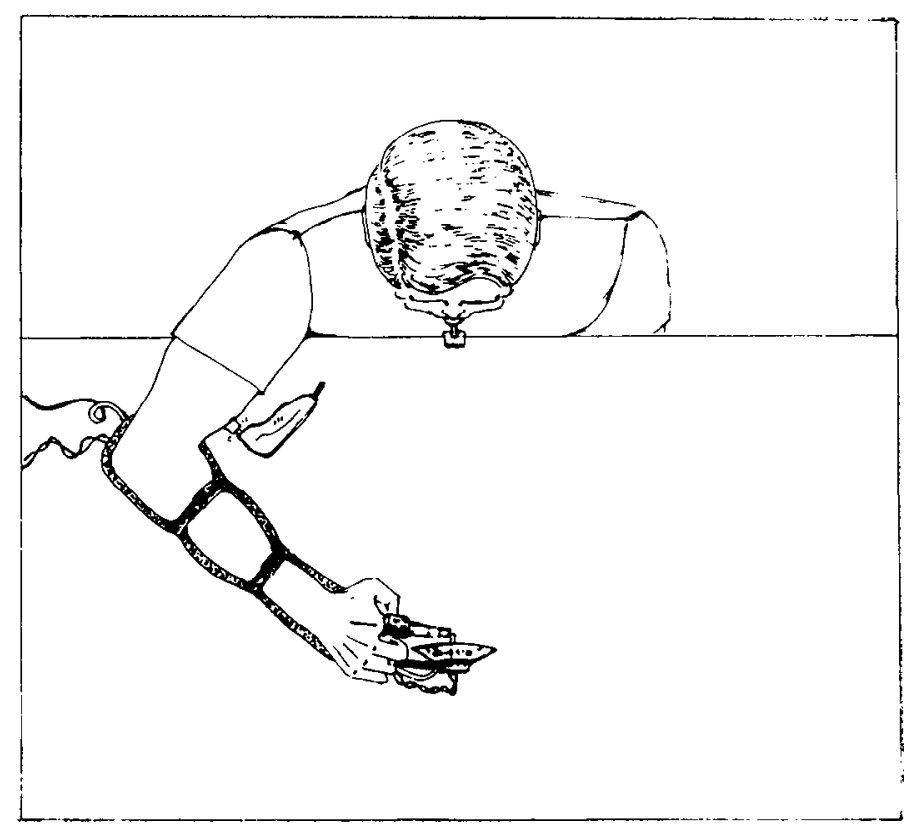

Figure 1. The subject's restrained forearm is oriented in a horizontal plane with the index finger positioned in the projection of the median plane of the head; the finger is in contact with a miniature auditory speaker and a fiberoptic strand. A biteplate stabilizes the head.

a $110^{\circ}$ angle in a vertical plane. In the other apparatus, the subject's right arm was also fixed at a $110^{\circ}$ angle but in a horizontal plane. In both apparatus, the tip of the subject's right index finger was at eye level and in the median plane of his head. Figure 1 illustrates the experimental situation for testing in the horizontal plane.

A fiberoptic strand, 20 mil in diameter, covered except for its tip, and a miniature audio speaker were attached to the subject's right index finger with surgical tape and were oriented to the midpoint of his interocular axis. Both were in actual physical contact with the finger. The fiberoptic strand was connected to a remote light source and, when illuminated, provided a photopically effective fixation target. This target was the only object ever visible to the subject during the experiment. The speaker was fed by a waveform generator and, when activated, emitted 1 -msec clicks at $85 \mathrm{~Hz}$ and $70 \mathrm{~dB}$ SPL

Procedure. All subjects participated in pre-experimental training sessions in which illusory motion of their forearm was elicited by vibration; they also practiced describing this motion and rating its extent in degrees of elbow joint change. The experimenter used a goniometer to demonstrate various elbow angle displacements of known size, and the subjects practiced duplicating and rating them. The subjects were also given training in estimating displacements of the auditory and visual targets by allowing them to move their hand with the target(s) attached, and by displacing the targets to different positions on the arc described by the motion of the arm and having the subjects rate the displacement of the target(s) in degrees of elbow angle.

After pretraining had been completed, each subject participated in two experimental sessions conducted on separate days. A session involved four 30-sec vibration conditions in one apparatus, a 15-min break, and four 30$\mathrm{sec}$ trials in the other apparatus. The four conditions were: (1) biceps brachil vibration with no targets, (2) biceps vibration with visual target, (3) biceps vibration with auditory target, and (4) biceps vibration with auditory and visual targets.

Apparatus and condition order were balanced across subjects to control for possible sequence effects. A hand-held physiotherapy vibrator, 120 pulses/sec, was used to stimulate the biceps brachii muscle during the trials. Vibration at this frequency is known to activate both primary and secondary spindle endings (Burke et al., 1976).

An experimental session began with a review of the experiment. Each condition incuded a $10-\mathrm{sec}$ pre-vibration period in which the subject was in position on the biteplate and attempting to fixate his unseen index finger; depending on the condition, the auditory and/or visual target were also present. The subject reported any experienced motion of hand or targets. Vibration then began. During the $30-\mathrm{sec}$ vibration period, the subject reported the onset of arm and/or target movement, paying great care to indicate the relative order of their apparent motion. The subject called out aloud the 
sequence of apparent motion by naming the elements of motion, e.g., "hand and light" to indicate that the two started moving together; latency was determined by measuring the elapsed time from the onset of vibration to the beginning of the named element in the continuous tape recording that was made of each condition. After the trial, the subject estimated in degrees of elbow angle the maximum perceived displacement of his hand and of any targets present. He also described the arm and target movements experienced during the trials and drew illustrations of these patterns. During the experimental sessions, eye movements were not recorded for all subjects because extensive investigation has shown that, in the absence of a visual target, the eyes will follow the changing apparent position of the hand; when a visual target is attached to the hand, the eyes will maintain steady fixation although the light is perceived as moving (Lackner and Levine, 1978, 1979, 1981; Levine and Lackner, 1979; Lackner and Taublieb, 1984). Eye movements were recorded from two of our subjects in all of their experimental conditions, using an infrared corneal reflection system, with an accuracy of resolution of $0.5^{\circ}$; the records were in accord with our earlier observations.

\section{Results}

Biceps vibration-Hand. In all trials, shortly after the onset of vibration, subjects reported forearm motion, rightward in the horizontal apparatus, downward in the vertical apparatus. The forearm was felt to achieve a particular maximum extension and then to still be in motion but not displacing.

Biceps vibration-Hand and visual target. In $85 \%$ of all trials, shortly after or coincident with apparent motion of the forearm, visual motion in the same direction was reported. Like the arm, the visual target seemed to reach a peak displacement and then to still be moving but not displacing. No changes in apparent head position were reported but subjects said that their eyes "tracked" their hand and the visual target.

Biceps vibration-Hand and auditory target. Auditory motion and displacement were reported in $88 \%$ of all trials shortly after or coincident with reports of apparent motion of the forearm, in the same direction and the same plane. Auditory motion continued to be experienced after apparent auditory displacement ceased. One subject, when being tested in the horizontal apparatus, reported an apparent deviation of his head in the direction of experienced auditory (and forearm) displacement, and by a comparable amount.

Biceps vibration - Hand and auditory and visual targets. Auditory and visual motion and displacement were reported as accompanying or soon following apparent movement of the forearm in $91 \%$ and $85 \%$, respectively, of all trials. The forearm, the auditory target, and the visual target were perceived as achieving various maximum displacement while still seeming to move.

In all four experimental conditions after the apparent displace ments of the hand and auditory or visual target had reached their maximum extents, apparent motion continued until vibration was terminated. Apparent auditory or visual displacement never continued to increase in any of the trials after apparent displacement of the forearm had reached its greatest extent. Table I presents the

\section{TABLE}

Latencies (in seconds) after the onset of vibration of the right biceps brachii muscle before the first report of target movement

Ir] the horizontal test conditions, the direction of apparent motion was in a horizontal plane; for the vertical conditions, it was in the median plane of the body. The four test conditions were: (1) hand alone, (2) target light attached to the index finger, (3) sound source attached to the index finger (4) light and sound sources attached to the index finger. The experiment was conducted in darkness. Eight subjects participated.

\begin{tabular}{lllllllllll}
\hline & & \multicolumn{8}{c}{ Test Conditions } \\
\cline { 3 - 10 } & & Hand & $\begin{array}{c}\text { Hand- } \\
\text { Light }\end{array}$ & \multicolumn{2}{c}{$\begin{array}{c}\text { Hand- } \\
\text { Sound }\end{array}$} & $\begin{array}{c}\text { Hand-Light- } \\
\text { Sound }\end{array}$ \\
\hline \multirow{3}{*}{ Horizontal } & Mean & 2.3 & 3.2 & 4.1 & 3.6 & 4.4 & 3.2 & 3.4 & 4.1 \\
& Vertical & 1.2 & 2.3 & 3.1 & 2.4 & 3.3 & 1.9 & 2.0 & 2.4 \\
& Mean & 2.7 & 3.3 & 5.2 & 3.1 & 5.8 & 3.3 & 4.3 & 6.4 \\
& SD & 1.4 & 2.8 & 5.2 & 1.5 & 4.8 & 2.7 & 2.9 & 5.8 \\
\hline
\end{tabular}

TABLE ॥

Maximum apparent displacements (in degrees), specified in relation to the elbow joint, of hand and targets during vibration of the right biceps brachii muscle

The apparent displacements were always in the direction of elbow extension. In horizontal test conditions, the plane of apparent motion was horizontal; in vertical conditions, the place of apparent motion coincided with the median plane of the body. The test conditions were: (1) hand alone, (2) target light attached to the index finger, (3) sound source attached to the index finger, and (4) light and sound sources attached to the index finger. The trials were conducted in darkness. Eight subjects participated.

\begin{tabular}{|c|c|c|c|c|c|c|c|c|c|}
\hline \multirow[b]{3}{*}{ Horizontal } & \multirow[b]{3}{*}{$\begin{array}{l}\text { Mean } \\
\text { SD }\end{array}$} & \multicolumn{8}{|c|}{ Test Conditions } \\
\hline & & \multirow{2}{*}{$\begin{array}{r}\text { Hand } \\
34.9 \\
13.2\end{array}$} & \multicolumn{2}{|c|}{ Hand-Light } & \multicolumn{2}{|c|}{ Hand-Sound } & \multicolumn{3}{|c|}{ Hand-Light-Sound } \\
\hline & & & $\begin{array}{l}32.6 \\
12.9\end{array}$ & $\begin{array}{l}20.7 \\
12.7\end{array}$ & $\begin{array}{l}36.4 \\
15.4\end{array}$ & $\begin{array}{l}29.3 \\
14.5\end{array}$ & $\begin{array}{l}33.3 \\
17.6\end{array}$ & $\begin{array}{l}19.0 \\
14.0\end{array}$ & $\begin{array}{l}25.9 \\
18.6\end{array}$ \\
\hline Vertical & $\begin{array}{l}\text { Mean } \\
\text { SD }\end{array}$ & $\begin{array}{l}33.5 \\
14.2\end{array}$ & $\begin{array}{l}31.8 \\
13.4\end{array}$ & $\begin{array}{r}17.8 \\
8.7\end{array}$ & $\begin{array}{l}32.8 \\
16.2\end{array}$ & $\begin{array}{l}22.5 \\
12.5\end{array}$ & $\begin{array}{l}28.6 \\
17.2\end{array}$ & $\begin{array}{l}20.6 \\
11.7\end{array}$ & $\begin{array}{l}18.1 \\
10.4\end{array}$ \\
\hline
\end{tabular}

latencies, in seconds, of onset of arm and target(s) motion separately for the horizontal and vertical test situations. Table II presents the average target displacements in degrees. In the pre-vibration periods of the experimental conditions, none of the subjects reported apparent motion of their hand or of the auditory or visual target.

Correlated $t$ tests (one-tailed) were used to compare performance on different experimental days and under the horizontal and vertical apparatus test situations. No significant differences appeared; therefore, the data were combined for individual subjects across these dimensions. The latency and displacement scores for the four experimental conditions were then evaluated by an analysis of variance (ANOVA) and correlated $t$ tests. The onset of apparent motion of hand, light, and auditory target differed significantly $(p<$ 0.01 ); also, there was an interaction between experimental condition and latency of apparent movement of the targets $(p<0.005)$.

In all hand and target conditions, the onset of apparent hand movement always coincided with or preceded slightly the onset of apparent auditory or visual motion. Comparing across conditions, the latency of hand movement $(3.1 \mathrm{sec})$, and the latency of target light movement $(4.2 \mathrm{sec})$, the significance level is $p<0.005$ ); comparing latencies of hand and of auditory target motion (mean, $5.2 \mathrm{sec}$ ), the value is also $p<0.005$. In addition, the latency to apparent motion of the hand and arm was least in the hand-alone condition compared with the three conditions involving an auditory and/or visual target on the hand $(p<0.05)$.

An ANOVA evaluating the apparent displacement data indicated that average peak displacement was affected by condition $(\rho<$ 0.005 ) and was significantly different for the hand, light, and auditory targets. An interaction between conditions and targets was also present $(\rho<0.001)$. Correlated $t$ tests (one-tailed) showed that, across conditions, the mean apparent displacement of the hand $\left(33^{\circ}\right)$ was greater than that of the visual $\left(19.6^{\circ}\right)$ or the auditory target $\left(24.1^{\circ}\right)$ ( $p<0.005$ for both comparisons).

The subjective experiences described by the participants were similar. All of them reported seeing the target light move in the same direction as their arm was felt to be moving, either in pace with the arm or lagging behind. All reported hearing the sound move in a similar way in relation to their arm. None of the subjects reported auditory or visual motion that was not associated with apparent arm motion.

\section{Discussion}

Several features of the present experimental findings are notable in terms of how changes in auditory and visual localization relate to 
changes in apparent arm position. ${ }^{4}$ First, changes in the apparent spatial locations of the auditory and visual targets were always in the same direction and plane as the apparent displacement of the arm; second, illusory changes in arm position always preceded in time or coincided with changes in auditory and visual localization third, the magnitude of illusory arm displacement always exceeded or equalled that of the auditory and visual targets; fourth, all subjects experienced a dissociation of apparent velocity and displacement of arm and targel(s); the arm and the auditory and visual targets were perceived to attain peak apparent displacements and then seemed to still be in motion but not displacing.

This pattern indicates that vibration-induced changes in auditory and visual spatial localization were causally related to changes in apparent arm position. Vibration leads to abnormal patterns of spindle activity that are interpreted centrally as motion of the arm; this central representation of the arm is used in the perceptual assignment of the apparent directions of the visual and auditory targets attached to the hand. We know from studies of the oculobrachial illusion that if a visual target is attached to a subject's hand and he is instructed to fixate it, then his eyes will remain steadily fixating the target even though he sees it move in keeping with the apparent motion of his arm (Lackner and Levine, 1978; Levine and Lackner, 1979). In this situation, subjects will also spontaneously report feeling their eyes moving in the direction of the perceived displacement of the visual target. If their eyes were, in fact, centrally interpreted as in motion, this would explain why a retinally stable visual target was perceived as moving. However, such an explanation cannot account for the auditory motion experienced by subjects in the present study.

In this regard, it is relevant that only one subject reported during experienced horizontal auditory displacement a corresponding change in the apparent position of his head. For this subject, the change in auditory direction can be attributed at least in part to a changing representation of head position. However, for the remaining subjects, changes in auditory localization were not accompanied by perceptible changes in head position, and, because these auditory displacements were large $\left(20^{\circ}\right.$ or more), it is unlikely that subthreshold changes in apparent head position could be responsible. Instead, it appears that the spatial position assigned to an unchanging pattern of auditory cues at the ears, a pattern which is physically consistent only with a sound in the median plane of the head, can be influenced by brachial information about target location. Visual capture of sounds is, of course, a familiar phenomenon and is usually invoked to "explain" why voices are heard as emanating from the appropriate characters on a cinema screen or a television screen (Tastevin, 1937; Lackner, 1973; Welch, 1978). In

\footnotetext{
${ }^{4}$ The findings that have been described represent our second evaluation of the influence of aberrant proprioceptive information on auditory and visual localization. In our first attempt, not only did we not find an effect on auditory localization but we failed to replicate our reports (Lackner and Levine, 1978, 1981; Levine and Lackner, 1979) of an oculobrachial illusion. Because these original findings had been extremely robust, we analyzed how the later test situation differed from the original ones. In the earlier studies, the subject's hand or fingers were in physical contact with the target light housing; in the failure to replicate, the target light and the auditory target were attached to the end of the arm holder a few centimeters from the subject's fingers but not in physical contact. We found that, when we attached the targets to the hand or fingers, then vibration-induced illusory motion of the arm reliably elicited changes in the seen and heard positions of the visual and auditory targets. This finding indicates that cognitive knowledge of target position in relation to the hand, e.g., targets attached to the arm holder, is not sufficient to allow apparent arm position to affect visual and auditory localization. In ongoing studies, we are finding that it is perceptual coincidence-not physical coincidence-of hand and targets that seems to be the critical factor (J. R. Lackner, manuscript in preparation).
}

the present case, however, a form of spatial biasing from the hand is occurring: one illusion is creating another.

The occurrence of this phenomenon indicates a tremendous lability in the spatial representation of sounds and parts of the body. In this context, the existence of multiple topographic sensory maps in the brain, the multiple visual, auditory, and somatosensory maps and their possible functions, comes to mind. It should not go unnoticed that there are multiple associative connections between these maps and other brain regions that receive polysensory inputs. Such regions provide potential sites for mechanisms by which multiple sources of afferent and efferent information about the ongoing configuration of the body can be interrelated with patterns of auditory, visual, somatosensory, and proprioceptive afference (cf. Mountcastle et al., 1975; Woolsey, 1981; Hyvarninen, 1982; Jay and Sparks, 1982; May, 1983). It is clear that the assignment of auditory and visual direction to external targets is not contingent solely on patterns of auditory and visual activation of modality-specific topographic maps but also involves nonauditory and nonvisual spatial information about the ongoing configuration of the entire body. Moreover, such influences are generally bidirectional; in the present study, for example, having either an auditory or a visual target on the hand significantly increased the latency for induction of illusory arm motion by vibration.

In summary, it is becoming increasingly obvious that the relationship between represented body orientation and the apparent spatial direction attributed to exteroceptive patterns of stimulation is dependent on highly complex bidirectional interactions implicating many sensory and motor systems of the body. We know from other experiments that this interaction even extends to the perceptual representation of volition. It is possible to create situations in which one perceives oneself to be making movements of directions, amplitudes, or frequencies that differ from those actually being voluntarily executed (cf. Lackner and Mather, 1981; DiZio and Lackner, 1982; Lackner and DiZio, 1984). Moreover, movement and orientation are dependent on dynamic adaptations to terrestrial background force levels. During voluntary locomotion while exposed to twice Earth-gravity force levels, subjects experience illusory motion of their bodies and the environment because of the altered relationship between voluntary commands to the musculature and the resulting movements of the body (Lackner and Graybiel, 1981). During exposure to free fall, in the absence of vision and somatosensory cucs, subjects often lose all direct sense of their position within their surroundings and remain aware only of the relative configuration of their bodies and of their environment (Lackner and Graybiel, 1979, 1983). Such findings in combination with our present observations point to the tremendous subtlety and complexity of the mechanisms subserving spatial orientation, sensory localization, and movement.

\section{References}

Burke, D., K. -E. Hagbarth, L. Lofstedt, and B. G. Wallin (1976) The responses of human muscle spindle endings to vibration of non-contracting muscles. J. Physiol. (Lond.) 261: 673-694

Burgess, P. R., and F. J. Clark (1969a) Dorsal column projection of fibers from the cat knee joint. J. Physiol. (Lond.) 203: 301-315.

Burgess, P. R., and F. J. Clark (1969b) Characteristics of knee joint receptors in the cat. J. Physiol. (Lond.) 203: 317-335.

Clark, F. J., and P. R. Burgess (1975) Slowly adapting receptors in the cat knee joint: Can they signal joint angle? J. Neurophysiol. 38: 1448-1463

DiZio, P., and J. R. Lackner (1982) Some visual, efferent, and substrate of support influences on the control and appreciation of movement and spatial stability. Soc. Neurosci. Abstr. 8: 949 .

Goodwin, G. M., D. I. McCloskey, and P. B. C. Matthews (1972a) The persistence of appreciable kinesthesia after paralysing joint afferents but preserving muscle afferents. Brain Res. 37: 326-329.

Goodwin, G. M., D. I. McCloskey, and P. B. C. Matthews (1972b) Proprioceptive illusions induced by muscle vibration: Contribution to perception by muscle spindles? Science 175: 1382-1384.

Goodwin, G. M. D. I. McCloskey, and P. B. C. Matthews (1972c) The contribution of muscle afferents to kinaesthesia shown by vibration in- 
duced illusions of movement and by the effects of paralysing joint afferents. Brain 95: 705-748.

Granit, R. (1972) Constant errors in the execution and appreciation of movement. Brain 95: 649 .

Graybiel, A., and D. I. Hupp (1946) The oculogyral illusion: A form of apparent motion which may be observed following stimulation of the semicircular canals. J. Aviat. Med. 17: 3-27.

Graybiel, A., and J. Niven (1951) The effect of a change in direction of resultant force on sound localization: The audiogravic illusion. J. Exp. Psychol. 42: 227-230.

Hagbarth, K. -E., and G. Eklund (1966) Motor effects of vibratory muscle stimuli in man. In Muscular Afferents and Motor Control, R. Granit, ed., pp. 177-186, Almqvist and Wiksell, Stockholm.

Hyvarninen, J. (1982) Posterior parietal lobe of the primate brain. Physiol. Rev. 62: 1060-1129.

Jay, M. F., and D. L. Sparks (1982) Auditory and saccade-related activity in the superior colliculus of the monkey. Soc. Neurosci. Abstr. 8: 951.

Lackner, J. R. (1973) Visual rearrangement affects auditory localization. Neuropsychologia 11: 29-32.

Lackner, J. R. (1983) Influence of posture on the spatial localization of sound. J. Audio Eng. Soc. 31: 650-661.

Lackner, J. R., and P. DiZio (1984) Some efferent and somatosensory influences on body orientation and oculomotor control. In Sensory Experience, Adaptation and Perception, L. Spillmann and B. R. Wooten, eds., in press, Erlbaum Associates, Hillsdale, CA.

Lackner, J. R., and A. Graybiel (1978a) Postural illusuions experienced during $Z$-axis recumbent rotation and their dependence on somatosensory stimulation of the body surface. Aviat. Space Environ. Med. 49: 484-488.

Lackner, J. R. and A. Graybiel (1978b) Some influences of touch and pressure cues on human spatial orientation. Aviat. Space Environ. Med. 49: $798-804$.

Lackner, J. R., and A. Graybiel (1979) Parabolic flight: Loss of sense of orientation. Science 206: 1105-1108.

Lackner, J. R., and A. Graybiel (1981) Illusions of postural, visual, and aircraft motion elicited by deep knee bends in the increased gravitoinertial force phase of parabolic flight. Exp. Brain Res. 44: 312-316.

Lackner, J. R., and A. Graybiel (1983) Perceived orientation in free fall depends on visual, postural, and architectural factors. Aviat. Space Environ. Med. 54: 47-51.

Lackner, J. R., and M. S. Levine (1978) Visual direction depends on the operation of spatial constancy mechanisms. Neurosci. Lett. 7: 207-212.

Lackner, J. R., and M. S. Levine (1979) Changes in apparent body orientation and sensory localization induced by vibration of skeletal muscles: Vibratory myesthetic illusions. Aviat. Space Environ. Med. 50: 346-354.

Lackner, J. R., and M. S. Levine (1981) The guidance of saccadic eye movements to perceptually mislocalized visual and non-visual targets. Aviat. Space Environ. Med. 52: 461-465.

Lackner, J. R., and J. A. Mather (1981) Eye-hand tracking using afterimages: Evidence that sense of effort is dependent on spatial constancy mechanisms. Exp. Brain Res. 44: 138-142.

Lackner, J. R., and A. B. Taublieb (1984) Influence of vision on vibrationinduced illusions of limb movement. Exp. Neurology 85: 97-106.

Levine, M. S. and J. R. Lackner (1979) Some sensory and motor factors influencing the control and appreciation of eye and limb position. Exp. Brain Res. 36: 275-283.

Matthews, P. B. C. (1972) Mammalian Muscle Receptors and their Central Actions, Edward Arnold (Publishers) Ltd., London.

Matthews, P. B. C. (1982) Where does Sherrington's "muscular sense" originate? Muscles, joints, corollary discharges? Annu. Rev. Neurosci. 5 . 189-218.

McCloskey, D. I. (1978) Kinesthetic sensibility. Physiol. Rev. 58: 763-820.

McCloskey, D. I. (1981) Corollary discharges: Motor commands and perception. In Handbook of Physiology. Sect 1: The Nervous System. Vol. Il: Motor Control, V. B. Brooks, ed., pp. 1415-1447, American Physiological Society, Belhesda.

Mountcastle, V. B., J. C. Lynch, A. Georgopoulos, H. Sakata, and C. Acuna (1975) Posterior parietal association cortex of the monkey: Command functions for operations within extrapersonal space. J. Neurophysiol. 38 : $871-908$.

Sherrington, C. S. (1900) The muscular sense. In Textbook of Physiology, E. A. Schafer, ed., Vol. 2, pp. 1002-1025, Pentaland, Edinburgh.

Sherrington, C. S. (1906) On the proprio-ceptive system, especially in its reflex aspects. Brain 29: 467-482.

Sparks, D. L., and L. E. May (1983) Role of the monkey superior colliculus in the spatial localization of saccade targets. In Spatially Oriented Behavior, A. Hein and M. Jeannerod, eds., pp. 63-85, Springer-Verlag, New York.

Tastevin, J. (1937) En partant de l'experience d'Aristole. L'Encephale 1:57$84,140-158$

von Bekesy, G. (1960) Experiments in Hearing. McGraw Hill Book Co., New York.

Wallach, H. (1939) On sound localization. J. Acoust. Soc. Am. 10: 270-274.

Wallach, $H$. (1940) The role of head movements and vestibular and visual cues in sound localization. J. Exp. Psychol. 27: 339-368.

Welch, R. (1978) Perceptual Modification: Adapting to Altered Sensory Environments, Academic Press, Inc., New York.

Woolsey, C. N. (1981) Cortical Sensory Organization, Humana Press, Clifton, NJ. 